\section{Propuesta para actualizar los contenidos curriculares de un curso universitario impartido en la Facultad de Odontología, Universidad de Costa Rica}

\author{
Proposal to update the curricular contents \\ of a university course taught at the Faculty \\ of Dentistry, University of Costa Rica
}

Tony Sánchez-Achío 1,a

${ }^{1}$ Universidad de Costa Rica, Facultad de Odontología, San José, Costa Rica.

a Máster en Ortodoncia.

\section{Correspondencia:}

Tony Sánchez-Achío: dr_tsanchez@hotmail.com 520-1100. Tibás, San José. Costa Rica ORCID: 0000-0002-2641-1102

\section{Editora:}

María Angélica Alvarez-Páucar

Universidad Nacional Mayor de San Marcos, Perú.

Conflicto de intereses: el autor declara no tener conflictos de interés.

Fuente de financiamiento: autofinanciado.

Recibido: $15 / 03 / 21$

Aceptado: $13 / 07 / 21$

Publicado: 05/10/21

\begin{abstract}
Resumen
Objetivo. Realizar una actualización de los contenidos curriculares para el programa del curso de la Licenciatura en Odontología: Crecimiento Facial y Desarrollo de la Oclusión, impartido por el Departamento de Odontopediatría y Ortodoncia, Facultad de Odontología, Universidad de Costa Rica, para el año 2019. Métodos. Se estudiaron los programas curriculares del curso Crecimiento facial y desarrollo de la oclusión del año 2014 al 2018, impartido por la Facultad de Odontología de la Universidad de Costa Rica; así mismo los programas de Licenciatura en Odontología de las universidades privadas que imparten esta carrera. Posteriormente, se realizó una lista con todos los temas referentes a la materia y se realizó una encuesta a especialistas en el campo, para definir la importancia de enseñarlos en el curso estudiado. Resultados. A partir de los datos obtenidos de las opiniones de los docentes, se enumeraron en orden descendente la importancia de los temas a integrar en el currículo del curso Crecimiento facial y desarrollo de la oclusión. Conclusión. Se logra realizar una propuesta educativa para actualizar los contenidos curriculares de un curso universitario, eliminando la subjetividad de un sólo profesor. Este tipo de consensos son necesarios para satisfacer las necesidades y demandas actuales de los futuros profesionales en esta área.
\end{abstract}

Palabras clave: Enseñanza; Currículo; Odontología (fuente: DeCS BIREME). 
curricular contents of a university course, eliminating the subjectivity of a single teacher. This type of consensus is necessary to satisfy the current needs and demands of the future professionals in this area.

Keywords: Teaching; Curriculum; Dentistry (source: MeSH NLM).

\section{Introducción}

Desde su inauguración en 1942, la Facultad de Odontología de la Universidad de Costa Rica ha buscado adaptarse a las necesidades de la sociedad costarricense y a la evolución natural de la odontología, sometiéndose a procesos de mejoramiento, siendo el plan de estudios de la carrera evaluado por diferentes procesos de revisión y reestructuración. Tal compromiso ha sido reconocido por entes nacionales e internacionales, otorgándose la acreditación por el Sistema Nacional de Acreditación de la Educación Superior en el $2009^{1-4}$.

El modelo curricular de la carrera de Licenciatura en Odontología es un híbrido entre el modelo innovador y el modelo norteamericano. Del modelo innovador se destacan programas preventivos, contenidos epidemiológicos y una organización administrativa departamental orientada hacia la docencia y el servicio. Con respecto al modelo norteamericano, se introducen las asignaturas del área básica y médica en los primeros años de la carrera, posteriormente las clínicas organizadas por especialidad, además de algunas asignaturas que conservan un enfoque curativo y rehabilitador ${ }^{5-6}$. El plan de estudios de la carrera de Licenciatura en Odontología de la Universidad de Costa Rica, está distribuido en 6 años, de los cuales los primeros 4 años se llevan cursos teóricos, laboratorios, talleres y prácticas preclínicas, para dejar los últimos 2 años con las prácticas clínicas propias de la profesión.

El curso Crecimiento Facial y Desarrollo de la Oclusión, cuya sigla es O-3002, es el primer curso del Departamento de Odontopediatría y Ortodoncia, tiene como requisito el curso de Anatomía Cabeza y Cuello (MN0100), se imparte los segundos semestres de cada año a los estudiantes de tercer año, constituyendo las bases teóricas para los cursos teóricos de Odontopediatría I y II, y Ortodoncia I y II; cursos teóricos de cuarto año de carrera. Esos cursos se complementarán posteriormente con los cursos Clínica de Odontopediatría y Ortodoncia I y II ${ }^{7}$. Este curso consta de una lección magistral de dos horas por semana, en la cual se desarrollan los contenidos teóricos que permiten al estudiantado en Odontología, cumplir con el objetivo del curso ${ }^{7}$, el cual consiste en conocer los conceptos y procesos del crecimiento y desarrollo humano normal y/o patológico, con el fin de ser aplicados en el diagnóstico y tratamiento de las alteraciones relacionados con el complejo cráneo-facial.

El contenido curricular o currículo de un curso se refiere a los elementos que conforman una propuesta como respuesta a un conjunto de intenciones educativas, elaborado por diferentes sectores sociales ${ }^{7-10}$. La necesidad de realizar una actualización del contenido curricular responde al logro de los objetivos establecidos para el curso, que van cambiando con el tiempo a fin de cumplir las competencias laborales, concepciones y diseños deseados, logrando compenetrar al estudiante en diferentes campos del mercado mundial, cumpliendo con el perfil deseado del egresado ${ }^{11-15}$.

En la actualidad, la carrera de Licenciatura en Odontología es impartida por cuatro universidades costarricenses, Universidad de Costa Rica, Universidad Latina de Costa Rica, Universidad Latinoamericana de las Ciencias y la Tecnología, y Universidad Veritas. En la presente investigación se estudiaron los programas de todas las materias de la Licenciatura en Odontología de estas universidades, con el objetivo de realizar una actualización de los contenidos curriculares del curso Crecimiento Facial y Desarrollo de la Oclusión, impartido por el Departamento de Odontopediatría y Ortodoncia, Facultad de Odontología, Universidad de Costa Rica, para el año 2019.

\section{Métodos}

Esta investigación fue de naturaleza exploratoria, los objetivos a alcanzar no se han estudiado o abordado previamente, y presenta las bases para investigaciones futuras más complejas. El carácter fue mixto (cualitativo y cuantitativo), se comparó cualitativamente los planes de estudio de las diferentes universidades que imparten la Licenciatura en Odontología en el país, y cuantitativamente se realizó una encuesta numérica a diferentes especialistas en el campo, aplicando una lógica inductiva ${ }^{16}$.

Para iniciar la investigación, se analizaron los planes de estudio de la Licenciatura en Odontología, de la Universidad de Costa Rica y de las tres universidades privadas, facilitados por el Consejo Nacional de Enseñanza Superior Universitaria Privada (CONESUP). Al hacer el estudio de los contenidos curriculares que se ofrecen en las diferentes universidades, se observó que la única universidad que contiene un curso relacionado con el Crecimiento Facial y Desarrollo de la Oclusión, fue la Universidad de Costa Rica.

Por lo anterior, se realizó una lista con los contenidos del curso Crecimiento facial y desarrollo de la oclusión impartido por la Facultad de Odontología, Universidad de Costa Rica, durante el II semestre del $2018^{7}$ :

1. Definición de crecimiento, desarrollo y maduración. Métodos para medir el crecimiento.

2. Principios de genética humana. Alteraciones en la genética. 
3. Embriología y desarrollo de las estructuras periorales. Crecimiento facial prenatal y desarrollo del feto.

4. Embriología, desarrollo de la ATM y músculos masticatorios. Malformaciones del desarrollo.

5. Desarrollo dental y anomalías dentales. Desarrollo de la oclusión en dientes permanentes.

6. Teorías del control del crecimiento. Zonas y tipos de crecimiento del complejo cráneo-facial.

7. Influencias prenatales en el desarrollo facial. Dentición primaria y mixta. Secuencia y cronología de la erupción.

8. Dentición permanente. Patrones de crecimiento del complejo cráneo-facial. Maduración y envejecimiento.

9. Causas específicas de maloclusión. Influencias genéticas. Influencias ambientales.

10. Patrones de crecimiento en el paciente Clase I, II y III. Pacientes con el síndrome de la cara larga.

11. Evaluación de la edad esquelética. Interpretación de la radiografía carpal y vertebral.

12. Cronología en la modificación del crecimiento. Tratamientos ortopédicos en los 3 planos.

13. Principios de biomecánica orientados a ortopedia funcional.

14. Plan de tratamiento para los problemas esqueléticos en los preadolescentes y adolescentes.

15. Tratamiento para los problemas no esqueléticos (oclusales) en los preadolescentes y adolescentes.

Posteriormente, se realizó un estudio de los programas completos de la Licenciatura en Odontología de las tres universidades privadas, que no tienen un único curso destinado al crecimiento facial y desarrollo de la oclusión, pero si imparten cierto contenido en su malla curricular. Todo esto, con el objetivo de extraer los contenidos relacionados a este tema, analizando y comparando todas las materias y cursos que imparten. Para eso, tres observadores especialistas en el campo revisaron todos los planes de estudio, cabe destacar que estos especialistas son parte del cuerpo docente del Departamento de Odontopediatría y Ortodoncia de la Universidad de Costa Rica, por lo que también colaboraron en la encuesta final de esta investigación.

Se encontraron tres contenidos relacionados con el Crecimiento Facial y Desarrollo de la Oclusión, que las universidades privadas incluyen en sus planes de estudios, pero no son contemplados en el programa del curso Crecimiento Facial y Desarrollo de la Oclusión del $2018^{17-19}$ :

1. Introducción al diagnóstico de la mayoría de los problemas clínicos de ortodoncia, durante el período de la dentición temporal y mixta.
2. Etiología de la maloclusión, para enfocar mejor el diagnóstico, objetivos y plan de tratamientos de los pacientes.

3. Planos de los problemas que presenta el paciente, para establecer los objetivos y un plan de tratamiento adecuado para cada paciente.

Además, se analizaron los programas impartidos del curso Crecimiento Facial y Desarrollo de la Oclusión en la Universidad de Costa Rica en los últimos 4 años (20142017). En esta revisión se encontraron 2 temas que se eliminaron para el programa del curso del $2018^{20-23}$ :

1. Moldeado nasoalveolar en niños con labio y paladar hendido, distracción de mandíbula y maxila.

2. Maduración prenatal, neonatal y desarrollo de la función neuromuscular postnatal temprana.

Se realizó una lista final, con todos los temas mencionados anteriormente. Posteriormente se contó con 20 profesionales especialistas en el campo, 10 odontopediatras y 10 ortodoncistas, todos docentes de la Universidad de Costa Rica, quienes colaboraron resolviendo una encuesta, en la elección del contenido primordial a enseñar en el curso Crecimiento Facial y Desarrollo de la Oclusión y calificaron los temas teóricos según la importancia de contenerlos en dicho curso. La lista de temas a evaluar fueron del curso Crecimiento Facial y Desarrollo de la Oclusión del año 2018, así como los relacionados pero no contenidos en éste, tomados de los programas de las universidades privadas y de los programas de los últimos 4 años del mismo curso.

Se obtuvo una lista de 20 items (Tabla), en el cual los 20 profesionales expertos debían calificar los temas según la importancia de contenerlos en dicho curso, marcando con una equis de 0 a 2 y NS/NR, de la siguiente manera: 0: No incluir, 1: Incluir de forma moderada, 2: Importante incluir, NS/NR: No sabe / No responde.

\section{Resultados}

Los temas recopilados y relacionados al curso Crecimiento Facial y Desarrollo de la Oclusión, fueron enlistados para la consideración por parte de los docentes activos del Departamento de Odontopediatría y Ortodoncia durante el II Semestre del 2018. En la Tabla se muestran los resultados obtenidos, un total de 20 especialistas entre odontopediatras y ortodoncistas participaron en la encuesta.

A partir de estos valores absolutos, se pueden obtener los valores relativos para cada tema propuesto, como se muestra en la Figura 1. Este gráfico compara porcentualmente todas las opiniones de los temas que fueron evaluados por parte de los docentes activos del Departamento de Odontopediatría y Ortodoncia, Facultad de Odontología, Universidad de Costa Rica, durante el II semestre del 2018, para ser tomados en cuenta al actualizar y realizar el futuro programa del curso Crecimiento facial y desarrollo de la oclusión. 
Tabla. Opiniones por parte de los docentes del Departamento de Odontopediatría y Ortodoncia, acerca de los contenidos para el curso Crecimiento facial y desarrollo de la oclusión. U.C.R. 2018

\begin{tabular}{|c|c|c|c|c|}
\hline Tema & 0 & 1 & 2 & NS/NR \\
\hline 1. Definición de crecimiento, desarrollo y maduración. Métodos para medir el crecimiento. & 0 & 1 & 19 & 0 \\
\hline 2. Principios de genética humana. Alteraciones en la genética. & 0 & 9 & 11 & 0 \\
\hline 3. Embriología y desarrollo de las estructuras periorales. Crecimiento facial prenatal y desarrollo del feto. & 0 & 2 & 18 & 0 \\
\hline 4. Embriología, desarrollo de la ATM y músculos masticatorios. Malformaciones del desarrollo. & 0 & 1 & 19 & 0 \\
\hline 5. Desarrollo dental y anomalías dentales. Desarrollo de la oclusión en dientes permanentes. & 1 & 4 & 15 & 0 \\
\hline 6. Teorías del control del crecimiento. Zonas y tipos de crecimiento del complejo craneofacial. & 0 & 3 & 17 & 0 \\
\hline 7. Influencias prenatales en el desarrollo facial. Dentición primaria y mixta. Secuencia y cronología de la erupción. & 1 & 2 & 17 & 0 \\
\hline 8. Dentición permanente. Patrones de crecimiento del complejo craneofacial. Maduración y envejecimiento. & 2 & 1 & 17 & 0 \\
\hline 9. Causas específicas de maloclusión. Influencias genéticas. Influencias ambientales. & 1 & 3 & 16 & 0 \\
\hline 10. Patrones de crecimiento en el paciente Clase I, II y III. Pacientes con el síndrome de la cara larga. & 0 & 3 & 17 & 0 \\
\hline 11. Evaluación de la edad esquelética. Interpretación de la radiografía carpal y vertebral. & 0 & 6 & 14 & 0 \\
\hline 12. Cronología en la modificación del crecimiento. Tratamientos ortopédicos en los 3 planos. & 1 & 6 & 13 & 0 \\
\hline 13. Principios de biomecánica orientados a ortopedia funcional. & 3 & 12 & 5 & 0 \\
\hline 14. Plan de tratamiento para los problemas esqueléticos en los preadolescentes y adolescentes. & 5 & 10 & 5 & 0 \\
\hline 15. Tratamiento para los problemas no esqueléticos (oclusales) en los preadolescentes y adolescentes. & 7 & 5 & 8 & 0 \\
\hline 16. Moldeado nasoalveolar en niños con labio y paladar hendido, distracción de mandíbula y maxila. & 3 & 13 & 4 & 0 \\
\hline 17. Maduración prenatal, neonatal y desarrollo de la función neuromuscular postnatal temprana. & 2 & 10 & 8 & 0 \\
\hline 18. Diagnóstico de problemas clínicos de ortodoncia en dentición temporal y mixta. & 7 & 6 & 7 & 0 \\
\hline 19. Diagnóstico, objetivos y plan de tratamientos de los pacientes en dentición temporal y mixta. & 7 & 4 & 9 & 0 \\
\hline 20. Problemas del paciente, ubicación por planos, establecimiento de objetivos y planes de tratamiento. & 7 & 4 & 9 & 0 \\
\hline
\end{tabular}

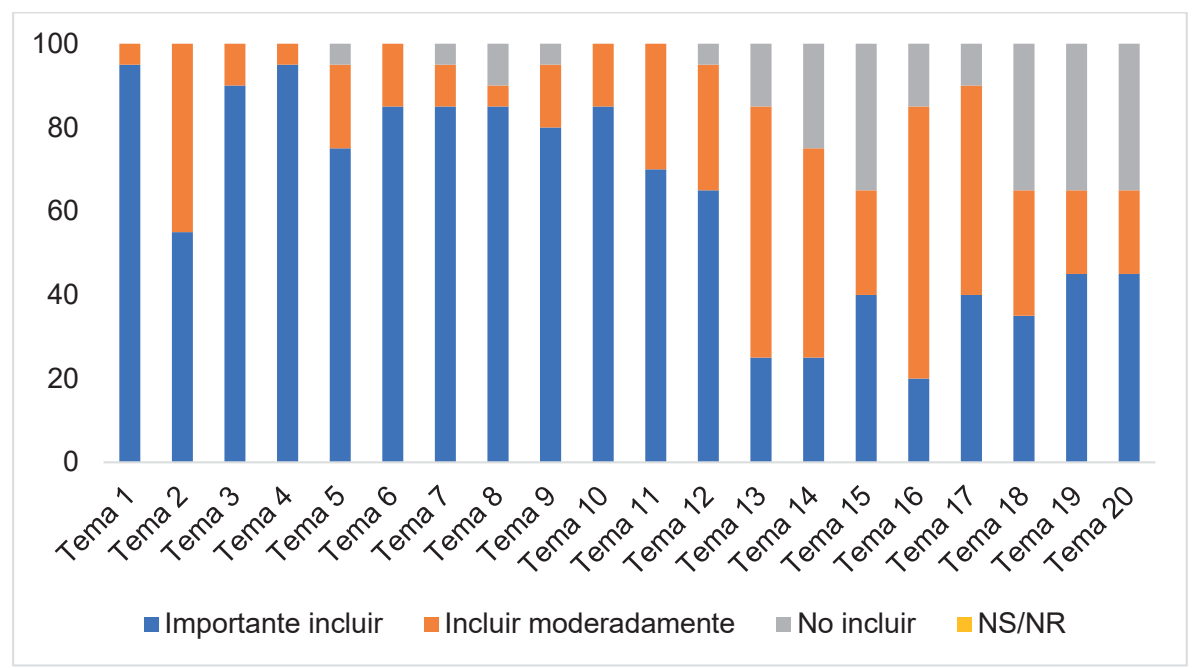

Figura 1. Comparación porcentual de la importancia de la inclusión de temas en el curso Crecimiento facial y desarrollo de la oclusión, según los docentes del Departamento de Odontopediatría y Ortodoncia. U.C.R. 2018 
Por último, se obtuvo un puntaje total para cada tema a partir de la valoración de los docente, si había seleccionado la casilla de "importante incluir" se le atribuía 2 puntos, "incluir moderadamente" 1 punto, o "no incluir" sin puntos. La Figura 2 nos muestra estos datos.

A partir de los datos obtenidos por las opiniones de los docentes, se pueden enumerar en orden descendente, la importancia de los temas a integrar en el currículo del curso Crecimiento Facial y Desarrollo de la Oclusión para el siguiente año (2019):

1. Definición de crecimiento, desarrollo y maduración. Métodos para medir el crecimiento.

2. Embriología, desarrollo de la ATM y músculos masticatorios. Malformaciones del desarrollo.

3. Embriología y desarrollo de las estructuras periorales. Crecimiento facial prenatal y desarrollo del feto.

4. Teorías del control del crecimiento. Zonas y tipos de crecimiento del complejo craneofacial.

5. Patrones de crecimiento en el paciente Clase I, II y III. Pacientes con el síndrome de la cara larga.

6. Influencias prenatales en el desarrollo facial. Dentición primaria y mixta. Secuencia y cronología de la erupción.

7. Dentición permanente. Patrones de crecimiento del complejo cráneo-facial. Maduración y envejecimiento.

8. Causas específicas de maloclusión. Influencias genéticas. Influencias ambientales.

9. Desarrollo dental y anomalías dentales. Desarrollo de la oclusión en dientes permanentes.

10. Evaluación de la edad esquelética. Interpretación de la radiografía carpal y vertebral.

11. Cronología en la modificación del crecimiento. Tratamientos ortopédicos en los 3 planos.
12. Principios de genética humana. Alteraciones en la genética.

13. Maduración prenatal, neonatal y desarrollo de la función neuromuscular postnatal temprana.

14. Principios de biomecánica orientados a ortopedia funcional.

15. Diagnóstico, objetivos y plan de tratamientos de los pacientes en dentición temporal y mixta.

16. Problemas del paciente, ubicación por planos, establecimiento de objetivos y planes de tratamiento.

17. Tratamiento para los problemas no esqueléticos (oclusales) en los preadolescentes y adolescentes.

18. Moldeado nasoalveolar en niños con labio y paladar hendido, distracción de mandíbula y maxila.

19. Plan de tratamiento para los problemas esqueléticos en los preadolescentes y adolescentes.

20. Diagnóstico de problemas clínicos de ortodoncia en dentición temporal y mixta.

En base a esta lista y conociendo que el ciclo lectivo en la Universidad de Costa Rica, consta de 16 semanas, dos para evaluaciones y 14 para impartir lecciones; la actualización curricular en esta investigación, consistiría en un programa que contenga los 14 temas más puntuados por los docentes del Departamento de Odontopediatría y Ortodoncia.

\section{Discusión}

Páez, resalta la importancia en la actualización de los contenidos curriculares, la cual es una estrategia comúnmente utilizada por docentes y especialistas curriculares en diferentes áreas del conocimiento, teniendo en cuenta las competencias profesionales necesarias en el ámbito laboral ${ }^{24}$.

Como se analiza en la Tabla, los temas en los cuales más docentes respondieron importante incluir en el temario

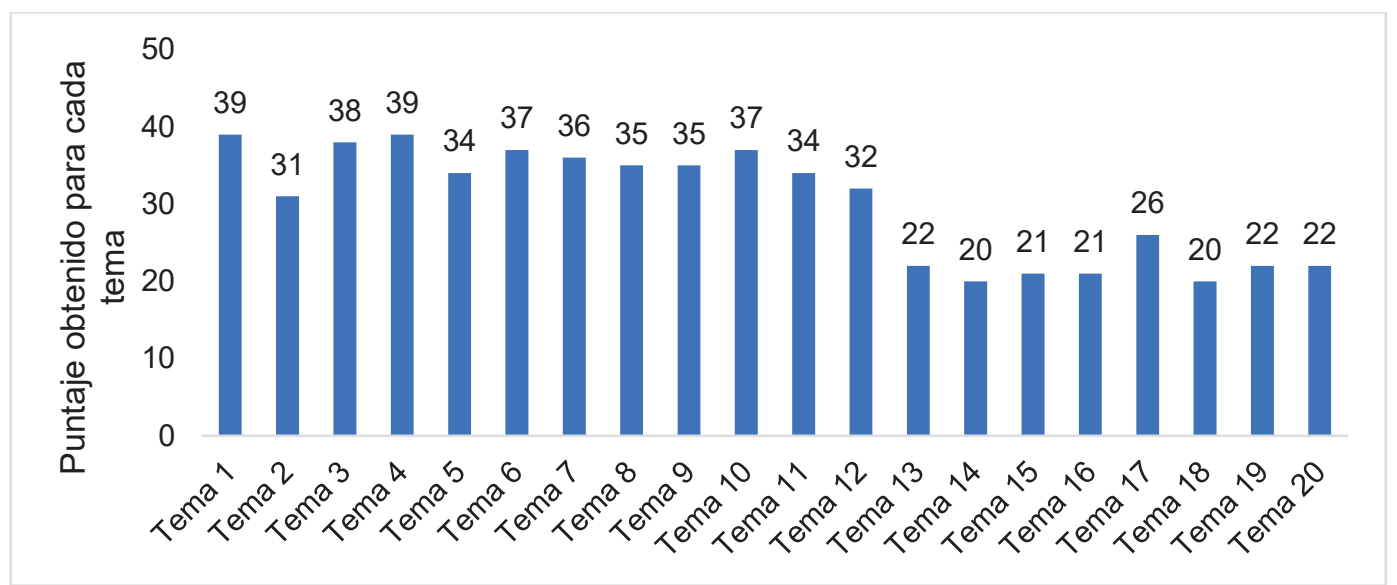

Figura 2. Comparación del puntaje acerca de la importancia de la inclusión de temas en el curso Crecimiento facial y desarrollo de la oclusión, según los docentes del Departamento de Odontopediatría y Ortodoncia. 
del curso Crecimiento Facial y Desarrollo de la Oclusión fueron: "Definición de crecimiento, desarrollo y maduración", "Embriología, desarrollo de la ATM y músculos masticatorios" y "Embriología y desarrollo de las estructuras periorales". Todos los temas obtuvieron en su mayoría una calificación de "importante", a excepción de los temas: "Moldeado nasoalveolar en niños", "Principios de biomecánica", "Plan de tratamiento para los problemas esqueléticos" y "Maduración prenatal, neonatal y desarrollo de la función neuromuscular postnatal temprana"; los cuales obtuvieron una calificación que los ubicaba en moderadamente importante de incluir en el programa del curso.

Por último, los temas menos aceptados para su inclusión (aunque fueron mayormente evaluados como importantes) fueron: "Tratamiento para los problemas no esqueléticos", "Diagnóstico de problemas clínicos de ortodoncia en dentición temporal y mixta", "Diagnóstico, objetivos y plan de tratamientos de los pacientes en dentición temporal y mixta" y "Problemas del paciente, ubicación por planos, establecimiento de objetivos y planes de tratamiento".

En cuanto, al estudio curricular del programa Crecimiento Facial y Desarrollo de la Oclusión, se pudo observar la necesidad e importancia de realizar una actualización para este curso, integrando la metodología y el currículo, contemplando la opinión de varios especialistas en el área; ya que desde el 2015, cuando el investigador toma la coordinación del curso, no se había realizado dichas actualizaciones formalmente. Se concuerda con los especialistas en el área de la docencia, sobre la necesidad de realizar periódicamente un estudio del contenido curricular para todos los cursos que se imparten en las universidades, con el fin de brindar programas actualizados acorde con la formación humanística integral, fortaleciendo la destreza, la habilidad, la opinión crítica, pero sobretodo las responsabilidades, paralelas a las necesidades que se requieren para formar un profesional competente con la realidad actual ${ }^{8-10,12,14-16}$.

Al revisar los programas curriculares de la Licenciatura en Odontología que ofrecen todas las universidades en Costa Rica, se puede observar que la Universidad de Costa Rica, es la única que cuenta con un curso completo sobre el crecimiento facial y los cambios en la oclusión; por lo que se recomienda la integración de este curso dentro de sus planes de estudio, ya que es la base para el buen entendimiento de los cursos de odontopediatría y ortodoncia. Lo anterior, constituyó la principal limitación de esta investigación, y para solventarlo, se estudiaron los contenidos curriculares de la Licenciatura en Odontología de las universidades privadas que la imparten, con el fin de analizar los temas pertinentes al curso en estudio.

Como conclusión, se logra realizar una propuesta educativa para actualizar los contenidos curriculares de un curso universitario, eliminando la subjetividad de un sólo profesor. Se recomienda validar esta propuesta y realizar más estudios al respecto, que entre sus ventajas es el consenso de diferentes docentes para satisfacer las necesidades y demandas actuales de los futuros profesionales en las distintas áreas, sin embargo, para fortalecer la propuesta educativa es conveniente considerar además la evaluación de los contenidos partiendo del análisis de las competencias u objetivos del curso tal como señala Páez ${ }^{24}$.

\section{Referencias bibliográficas}

1. Ortiz JA. La pintoresca historia de la Escuela de Odontología. En Universidad de Costa Rica, Facultad de Odontología. Fac Odontol Univ, 1942-2002: 60 aniversario. 2002;1(1):11-24.

2. Hernández P. Antecedentes históricos de la Educación en Costa Rica: de la colonia a la apertura de la Facultad de Odontología, Universidad de Costa Rica. ODOVTOSInt J Dental Sc. 2013;15(1):81-91.

3. Ruiz A. La educación superior en Costa Rica. San José, Costa Rica: Editorial de la Universidad de Costa Rica. 2000. [Consultado el 10 de noviembre del 2018]. Disponible en: http://www.centroedumatematica.com/ aruiz/libros/La\%20Educacion\%20Superior\%20en\%20 Costa\%20Rica.pdf

4. Montero MP. Una mirada a la Facultad de Odontología en su 75 Aniversario. ODOVTOS- Int J Dental Sc. 2017;19(1):17-22.

5. Howard M, Jiménez MM. La reforma curricular de la carrera de Licenciatura en Odontología en el contexto del proceso de autoevaluación. Odovtos. 2009;11(1):26-35.

6. Sanz T. Modelos curriculares. Revista Pedagogía Universitaria. 2004;9(2):55-68.

7. Sánchez T. Programa del curso Crecimiento Facial y Desarrollo de la Oclusión. San José, Costa Rica: Universidad de Costa Rica, Facultad de Odontología; 2018.

8. Ávila G, Huerta ML, Cruz AG. (2019). Física II con enfoque sustentable: propuesta de actualización del programa de estudios en el bachillerato del IPN. Debates en Evaluación y Currículum/ Congreso Internacional de Educación: Currículum. [Consultado el 10 de noviembre del 2018]. Disponible en: https://posgradoeducacionuatx.org/pdf2019/A009.pdf

9. Hernández J, Botero H, Correa R. (2016). Aplicación de mapas conceptuales en la gestión de un programa curricular de educación superior en ingeniería. Concept Maps: Theory, Methodology, Technology Proc. of the Second Int. Conference on Concept Mapping. [Consultado el 10 de noviembre del 2018]. Disponible en: http:// cmc.ihmc.us/cmc2006Papers/cmc2006-p59.pdf

10. Molina MR, Camargo MJ, Gaxiola MT, Hamed AA. Implicaciones de la flexibilidad curricular en el programa educativo de derecho. Un estudio de caso. Rev investig académica front. 2019;12(31):1-33.

11. Icarte GA, Labate HA. Metodología para la revisión y actualización de un diseño curricular de una carrera universitaria incorporando conceptos de aprendizaje basado en competencias. Form Univ. 2016;9(2):3-16.

12. Pacheco A. Análisis, revisión y actualización del programa analítico de la asignatura inteligencia artificial [Trabajo de 
Grado]. [Cuba]: Departamento de Inteligencia Artificial, Universidad de Oriente; 2016.

13. Quijano M. Propuesta de actualización curricular del programa ingeniería industrial. [Trabajo de Grado]. [Colombia]: Facultad de Ingeniería, Universidad Autónoma de Occidente; 2018.

14. Silva MP, Alves M. Um currículo na integração ensino-serviço do programa mais médicos e possíveis efeitos culturais. Trab Educ Saúde. 2019;17(3):1-18.

15. Gómez VM, Celis JE. Factores de innovación curricular y académica en la educación superior. Revista ieRed. 2005;1(2):1-13.

16. Hernández R, Fernández C, Baptista P. Metodología de la investigación. México: Mc Graw Hill; 2014.

17. ULACIT Universidad Latinoamericana de las Ciencias y la Tecnología. Solicitud para Aprobar la Modificación de la Licenciatura en Odontología, a tenor del inciso c), del artículo 23, del Reglamento General del CONESUP. San José, Costa Rica: ULACIT; 2012.

18. Universidad Latina de Costa Rica. Cambios de ubicación de cursos en el plan de estudios, cambios de requisitos y actualización de contenidos en los programas de los cursos en la Licenciatura en Odontología. San José, Costa Rica: Universidad Latina de Costa Rica; 2008.
19. Universidad Veritas. Diseño curricular de la carrera Licenciatura en Odontología. San José, Costa Rica: Facultad Autónoma de Ciencias Odontológicas; 1995.

20. Sáenz I. Programa del curso Crecimiento Facial y Desarrollo de la Oclusión. San José, Costa Rica: Universidad de Costa Rica, Facultad de Odontología; 2014.

21. Sánchez T. Programa del curso Crecimiento Facial y Desarrollo de la Oclusión. San José, Costa Rica: Universidad de Costa Rica, Facultad de Odontología; 2015.

22. Sánchez T. Programa del curso Crecimiento Facial y Desarrollo de la Oclusión. San José, Costa Rica: Universidad de Costa Rica, Facultad de Odontología; 2016.

23. Sánchez T. Programa del curso Crecimiento Facial y Desarrollo de la Oclusión. San José, Costa Rica: Universidad de Costa Rica, Facultad de Odontología; 2017.

24. Páez H, Zabala V, Zamora R. Análisis y actualización del programa de la asignatura Automatización Industrial en la formación profesional de ingenieros electrónicos. Revista Educación en Ingeniería. 2016;11(21):39-44. 